\title{
Plasmonic photocatalytic reactor design: Use of multilayered films for improved organic degradation rates in a recirculating flow reactor
}

\author{
Jon W. Pickering, Venkat R. Bhethanabotla*, and John N. Kuhn* \\ University of South Florida. Tampa, FL 33620
}

\begin{abstract}
* To whom correspondence should be addressed: bhethana@usf.edu; (813) 974-3041 and jnkuhn@usf.edu; (813) 974-6498, fax n/a; Department of Chemical \& Biomedical Engineering, University of South Florida, Tampa FL 33620
\end{abstract}

\begin{abstract}
Plasmonic photocatalysis was investigated by examining both the arrangement of the photocatalyst and plasmonic components, and the structure and composition of the plasmonic phase. Pd was epitaxially grown on to $50 \mathrm{~nm} \mathrm{Ag}$ nanocubes. The $\mathrm{Ag}: \mathrm{Pd}$ ratio was optimized to blue-shift the plasmonic absorption peak to match the bandgap of $\mathrm{TiO}_{2}(\sim 405 \mathrm{~nm})$. A $\sim 5 \mathrm{~nm}$ Pd coating on the $\mathrm{Ag}$ nanocubes $(\mathrm{Ag}: \mathrm{Pd}=9: 1)$ led to the observation of the $\mathrm{Ag}$ nanocube's plasmonic feature. The arrangement of the photocatalyst components was tested for degradation of model organics in a slurry and a recirculating thin film photoreactor. The results demonstrated that both the arrangement of the components and the structure and composition of the plasmonic material influenced the conversion. The $\mathrm{TiO}_{2} / \mathrm{Ag}$ composite catalysts yielded slight improvement (in thin film reactor) or had a negative effect (slurry reactor) compared to $\mathrm{TiO}_{2}$ which was attributed to scattering light away from the semiconductor photocatalyst and/or covering some active sites. The addition of the Pd shell on the $\mathrm{Ag}$ nanocube yielded improved performance compared to the $\mathrm{TiO}_{2} / \mathrm{Ag}$ composite catalysts, likely due to electron trapping. A factor of 2 enhancement in rate and apparent quantum yield compared to $\mathrm{TiO}_{2}$ was achieved for the $\mathrm{Ag}$ $\mathrm{NC}$ layer underneath the $\mathrm{TiO}_{2}$ layer and was attributed to light scattering of absorbed photons. The addition of the $\mathrm{Pd}$ shell to the $\mathrm{Ag}$ nanocube still provided enhancement compared to $\mathrm{TiO}_{2}$, but was lesser compared to $\mathrm{Ag}$ layer due to lower scattering efficiency. The results of this study provide insights for plasmonic photocatalytic reactor design. Best utilization of plasmonic enhancement to photocatalysis is indicated via a layered design of the plasmonic and photocatalytic phases.
\end{abstract}

Keywords: reactor design; layered catalyst film; heterogeneous photocatalysis; plasmonic; titania 


\section{Introduction}

In recent years, employing advanced oxidation processes (AOPs) as a means of wastewater remediation has emerged as a promising route towards maintaining a sustainable global water management program. The heterogeneous photocatalytic oxidation process has been of particular interest due to the prospect of utilizing solar radiation as the driving force behind the degradation of pollutants. Of the photocatalysts studied to date, $\mathrm{TiO}_{2}$ remains the most attractive material for environmental applications due to its affordability, stability, biocompatibility, and relative high quantum yield. [1-4]. However, several key limitations of the heterogeneous photocatalytic oxidation process exist, which can be grouped into irradiation related (low and irregular photon flux in solar insolation), photocatalyst property related (large band gaps [5] and high charge carrier recombination rates [6]), and reactor design related (low photon and mass transfer rates [7]). Substantial efforts, mainly on the photocatalyst formulation [5, 8], have been expended to improve process performances. Reactor design has been advanced as well [7, 9], but to a lesser extent. The use of metal nanoparticles (NPs) for their optical, electrical, and catalytic functions are a common methodology to improve the performance of semiconductor photocatalysts.

Since Awazu et al [10] reported "plasmonic photocatalysis" in 2008, plasmonics has been an emerging phenomenon employed to improve the performance of catalysts. In subsequent studies, plasmon induced charge carriers [11-14], plasmonic heating [15], and chemical interface dampening [16] have been invoked to explain the enhancements, depending on the specific catalyst formulation, catalyst reaction, and conditions. However, these require high degrees of interfacial contact of the plasmonic phase to the semiconductor photocatalyst or the adsorbates for the enhancements to be realized. The same proximity situation is also required for electron trapping by metal doping and their use as co-catalysts [8, $10,13]$. Here, we will demonstrate that the scattering properties of a plasmonic phase can be optimized in reactor design to improve the performance of organic photodegradation reactors. 
The goal of this study is to examine photocatalytic reactor design for the degradation of organic species as a model AOP. We have designed and implemented multilayered films to improve the photocatalytic performance of traditional titania photocatalysts (Fig. 1). The plasmonic phase is employed to permit an intensified light field with wavelengths shorter than that of the wavelength associated with the semiconductors band gap $\left(\lambda \leq \lambda_{\mathrm{BG}}\right)$.

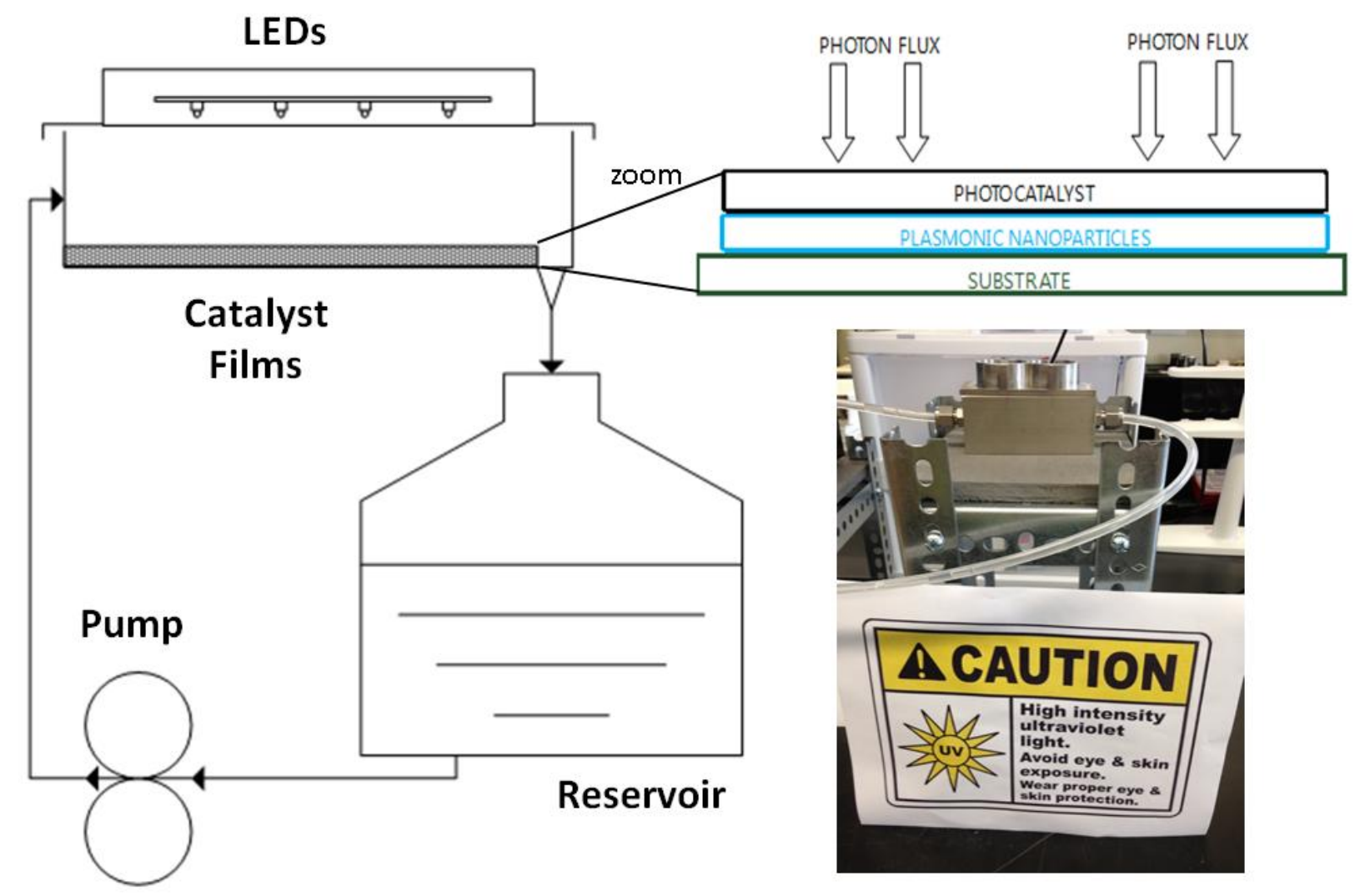

Fig. 1. Configuration of the titania+NP layered arrangement.

In this study, we aim to improve upon the experimental design of plasmon assisted photocatalysis by studying both the arrangement of the photocatalyst and plasmonic NP phase and by implementing an improved reactor setup that will subject thin film substrates to a recirculating, liquid phase environment. To achieve the differences in arrangement, a $\mathrm{TiO}_{2} / \mathrm{NP}$ composite was tested against a layered film arrangement in which a titania layer is deposited on a plasmonic NP layer, with both layers immobilized 
on a glass slide, which will be referred to as the $\mathrm{TiO}_{2}+\mathrm{NP}$ system as shown in Fig. 1 . The $\mathrm{TiO}_{2} / \mathrm{NP}$ composites were tested in a shallow dish batch reactor as well in the improved reactor by immobilizing it on a glass slide.

An additional aim of this study is to investigate compositional effects of the plasmonic phase. $\mathrm{Ag}$ nanocube (NC) cores were selected due to the easily tunable peak plasmonic resonance with cube side length [17]. However, since typical-sized NCs of $30 \mathrm{~nm}$ side length have plasmonic absorption peaks around $450 \mathrm{~nm}$, which is larger than the corresponding band gap of $\mathrm{TiO}_{2}(\sim 405 \mathrm{~nm})$, we attempted to lower the resonance wavelength by combining with $\mathrm{Pd}$, which is known to shift the plasmonic edge to lower values in Ag-Pd bimetallics [18, 19]. In addition to shifting the plasmonic resonance toward the $\mathrm{TiO}_{2}$ bandgap, this $\mathrm{Pd}$ shell may also protect the Ag core from oxidation, which is known to diminish plasmonic resonance. Ag oxidation was a primary concern of Awazu et al [10], though it is also worthwhile to mention that others have reported the highest performance improvement compared to $\mathrm{TiO}_{2}$ for low Ag loading in which oxidized $\mathrm{AgO}_{\mathrm{x}}$ species did form [20]. The Pd shell around the $\mathrm{Ag} \mathrm{NC}$ core would be a stable structure at the low temperatures of synthesis and application. At higher temperatures in vacuum, an alloy is predicted in Ag-rich formulations of Ag-Pd [21]. Although not with NC cores, stable Pd shells on Ag nanoparticles have been reported [22-24]. Challenges in synthesizing Ag-Pd NPs with Ag cores arise from the rather large lattice mismatch between $\mathrm{Ag}$ and $\mathrm{Pd}$ (4.6\%) and Ostwald ripening associated with the in situ growth of Pd on a Ag surface at elevated temperatures $[19,24]$. To avoid the latter issue, the Pd overgrowth occurred at much lower temperatures than that of the Ag NC synthesis in our work. In more reactive environments (higher temperature and/or more corrosive solvents), galvanic replacement occurs and results in hollow Pd boxes [25].

\section{Materials and methods}

\subsection{Metallic nanoparticle synthesis}

2.1.1. Ag nanocube (NC) synthesis 
The Ag NCs were synthesized using a polyol reduction method, following a scaled up version of that reported by Zhang et al.[17] Ethylene glycol (EG; $30 \mathrm{~mL}$; Sigma-Aldrich; 99\%) was added to a 250$\mathrm{mL}$ round bottom flask and heated to $150^{\circ} \mathrm{C}$ under magnetic stirring using an oil bath. After 30 min, a solution $\left(0.36 \mathrm{~mL}\right.$ ) of $3 \mathrm{mM}$ NaHS (Sigma-Aldrich; $\mathrm{NaSH} \cdot \mathrm{xH}_{2} \mathrm{O}$ ) in $\mathrm{EG}$ was injected into the flask. After $2 \mathrm{~min}$, solutions of $3 \mathrm{mM} \mathrm{HCl}$ (Sigma-Aldrich; 36.5-38.0\%; $3 \mathrm{~mL}$ ) and $7.5 \mathrm{~mL}$ of poly(vinyl pyrrolidone) (PVP; Alfa Aesar; $20 \mathrm{mg} / \mathrm{mL}$; MW = 58,000), both in EG, were injected into the flask. After another 2 min, $2.4 \mathrm{~mL}$ of $282 \mathrm{mM} \mathrm{CF}_{3} \mathrm{COOAg}$ (Sigma-Aldrich; 99.99\%) in EG was injected into the flask. During the entire process, the flask was capped except during the addition of reagents. Immediately following the addition of the $\mathrm{CF}_{3} \mathrm{COOAg}$ solution, vapors formed in the flask and the clear solution took on a yellowish color within $2 \mathrm{~min}$. This change in color indicated that the nucleation of Ag seeds had begun. The solution proceeded to shift colors from red, to reddish grey and finally to a greenish color with red, orange and blue undertones. After the desired time had passed from the addition of the $\mathrm{CF}_{3} \mathrm{COOAg}$, the flask was transferred to an ice water bath to quench the reaction. The Ag NCs were washed with acetone (VWR; reagent grade) once followed by 3 cycles of washing with ethanol (Sigma-Aldrich; 99.5\%) to remove excess PVP via centrifugation. The Ag NCs were then dispersed in ethanol for characterization and storage.

\subsubsection{Ag-Pd core-shell nanoparticle (NP) synthesis}

The Ag-Pd NPs were synthesized via a successive sequential reduction process in which Ag NCs were first synthesized as seeds following the previously described method. After quenching the reaction of $\mathrm{Ag} \mathrm{NC}$, the oil bath was cooled to a temperature of $90^{\circ} \mathrm{C}$ to inhibit Ostwald ripening and a solution containing an appropriate amount of $\mathrm{PdCl}_{2}$ (Sigma-Aldrich; 99.9) and $0.15 \mathrm{~g}$ of $\mathrm{PVP}(\mathrm{MW}=58,000)$ in $30 \mathrm{~mL}$ EG was prepared. A $1 \mathrm{~mL}$ sample was removed from the $\mathrm{Ag}$ seed solution once it reached room temperature to compare with the resultant Ag-Pd NPs. Upon reaching the desired temperature of $90^{\circ} \mathrm{C}$, the capped round bottom flask was returned back to the oil bath and the Pd precursor solution was added to the reaction mixture. Within the first couple of min, the solution darkened to a slight grey-green hue and then continued to darken to a deep blue-black color irrespective of the amount of Pd added. The 
reaction continued under magnetic stirring for $30 \mathrm{~min}$ and was then again quenched in an ice water bath. Excess capping agent was removed following the same 4 step washing process used for the Ag NCs. The Ag-Pd NPs were then dispersed in ethanol for characterization and storage.

\subsection{Catalyst preparation}

\subsection{1. $\mathrm{TiO}_{2} / \mathrm{NP}$ composites}

In a $20 \mathrm{~mL}$ scintillation vial, AEROXIDE ${ }^{\circledR} \mathrm{TiO}_{2} \mathrm{P} 25$ (Nippon Aerosil; 99.5\%; $0.4 \mathrm{~g}$ ) was dispersed in $10 \mathrm{~mL}$ of ethanol via sonication. Using a pipette, appropriate amounts of $\mathrm{Ag} \mathrm{NC}$ or $\mathrm{Ag}-\mathrm{Pd}$ NP solution were added to the titania/ethanol solution and were mixed via sonication for $10 \mathrm{~min}$. Depending on the application of the $\mathrm{TiO}_{2} / \mathrm{NP}$ composite, the ethanol was either allowed to evaporate and the $\mathrm{TiO}_{2} / \mathrm{NP}$ were washed once using ultrapure water (Direct-Q UV3 Millipore) or the solution was transferred to an airbrush (Paasche Airbrush Company) to be applied to microscope slides as a thin film using compressed air as the propellant.

\subsection{2. $\mathrm{TiO}_{2}+\mathrm{NP}$ films}

On a microscope slide, a layer of NPs was repeatedly deposited via solvent (ethanol) evaporation until a desired amount of NP was obtained on the slide. The resultant layer of NP was then covered by a layer of titania via the doctor blade method. The titania slurry for the doctor blade method was prepared by thoroughly mixing $0.4 \mathrm{~g} \mathrm{TiO}_{2}, 0.75 \mathrm{~mL}$ water, and $0.18 \mathrm{~mL}$ ethanol using mortar and pestle. The film depth was controlled by applying strips of packing tape to both side of the microscope slide, creating a sub $\mathrm{mm}$ thick trench into which the paste was evenly spread. The layered substrate was then subjected to air drying on a mechanical shaking table for $30 \mathrm{~min}$ to avoid cracking followed by $30 \mathrm{~min}$ in an oven at $100^{\circ} \mathrm{C}$.

\subsection{Material characterization}

UV-Vis spectroscopy was performed by a Perkin Elmer, Lambda $35 \mathrm{UV}-\mathrm{V}$ is spectrometer to analyze the extinction spectra of the Ag NCs and Ag-Pd NPs. The measurements were obtained in an ethanol suspension at equal densities (mass NP/volume solution). Images of the NP were taken using 
transmission electron microscopy (TEM) by a Philips, FEI Morgagni TEM to gain insight into particle size and shape distributions. The TEM copper grids were prepared by adding one drop of diluted NP solution in ethanol, and then vacuum dried at $40^{\circ} \mathrm{C}$ for $10 \mathrm{~min}$. TEM images of the TiO2 supported NPs were obtained with the Tecnai F20 microscope.

\subsection{Photocatalytic testing}

The photocatalytic activities of the $\mathrm{TiO}_{2} / \mathrm{NP}$ composites were measured under both UV and UVVis conditions. The UV-Vis experiments were conducted in a batch slurry reactor, which has been previously described [26] including a lack of photolysis under the conditions examined in this study. The slurry reactor was irradiated with five $8 \mathrm{~W}$ General Electric F8T5/D fluorescent bulbs (Color Rendering Index 75) which resulted in $60 \mathrm{~W} / \mathrm{m}^{2}$ (measured by a Li-Cor Pyranometer in the 400-100 $\mathrm{nm}$ region) at the liquid-air interface. Methyl orange (MO) was utilized as the model pollutant. The reaction volume was $100 \mathrm{~mL}$ and the catalyst loading was $1 \mathrm{~g}$ titania / L. Prior to light irradiation, MO was allowed to reach equilibrium by magnetic stirring of the slurry in the dark for $30 \mathrm{~min}$ which resulted in a supernatant dye concentration of $200 \mathrm{ppm}$ (using ultrapure water to dilute; $\mathrm{pH} \sim 7$ ). Aliquots $(1 \mathrm{~mL}$ ) were removed every $30 \mathrm{~min}$ and MO concentrations were measured by UV-Vis spectroscopy. Apparent quantum yields $\left(\Phi_{\mathrm{MO}}\right)$ for UV-Vis experiments were calculated using the experimentally determined degradation rates and photon flux as follows:

$$
\Phi_{M O}=\frac{R_{M O}\left[\frac{m o l}{S}\right]}{J\left[\frac{m o l}{m^{2} * s}\right] * A\left[m^{2}\right]} * 100 \%
$$

where $\mathrm{R}_{\mathrm{MO}}$ is initial degradation rate, $\mathrm{J}$ is photon flux, and $\mathrm{A}$ is the irradiated area.

The UV experiments were conducted in a recirculating batch reactor constructed from stainless steel and designed to have two LEDs mounted on top of a catalyst film. For the current study, two UV LEDs (Thorlabs; M405L2; $410 \mathrm{~mW}$ ) provided $405 \mathrm{~nm}$ light (13 nm FWHM). In these experiments, the 
portion of the fluid above the catalyst film is irradiated and the remainder of the fluid is recirculated using a pump. Two configurations of the $\mathrm{TiO}_{2}-\mathrm{NP}$ complex were studied in these trials. The first arrangement involved immobilizing a base NP layer on a glass slide and then coating the NP layer with a top layer of pure titania. The second arrangement involved a single layer of the $\mathrm{TiO}_{2} / \mathrm{NP}$ composite immobilized on a glass slide. Rose bengal (RB) dye was used as the model pollutant in the experiments with this reactor and UV-Vis spectroscopy was used to monitor the dye concentration as a function of time. The model organic species was changed due to the realization that, via the positive valence charge found in the MO structure, direct reduction by excited Ag NCs could interfere with the results. RB however has a neutral electronic state and direct reduction is less probable [27-30]. A total reaction volume of $100 \mathrm{~mL}$ was used with an initial $\mathrm{RB}$ dye concentration of $400 \mathrm{ppb}$ (using ultrapure water to dilute; $\mathrm{pH} \sim 7$ ) and $1 \mathrm{~mL}$ aliquots were removed every 5 or $15 \mathrm{~min}$ for concentration determination. In similar experiments, the decrease in total organic carbon (TOC) closely matched that of the model compound conversion [31]. Control experiments were conducted with the Ag nanocubes dispersed in a batch slurry reactor (at same $\mathrm{Ag}$ mass as in the slurry and recirculating batch reactors) and no RB conversion was measured.

\section{Results}

\subsection{Structural and optical characterization of Ag NCs and Ag-Pd NPs}

The extinction spectra (Fig. 2) of the NPs with various Ag:Pd ranging from 3:1 to 15:1 indicated that plasmonic properties of the $\mathrm{Ag}$ seeds were retained in $\mathrm{Ag}$-rich systems (Ag:Pd of 9:1 and greater). At a Ag:Pd of 3:1 (Fig. 2 (a)), a relatively horizontal extinction profile was obtained from the Ag-Pd NP. This result was caused the Pd shell thickness being over $3 \mathrm{~nm}$, which would be consistent with the distinctive black color of the solution. Previous studies have reported that the Ag core plasmonic features are quenched when the Pd shell is $\sim 2 \mathrm{~nm}$. [22, 24] With the 6:1 Ag:Pd sample, a small fluctuation in the extinction spectrum (Fig. 2 (b)) was observed from 326-423 nm, but there was no true peak or shape. Further decreases of Pd content relative to $\mathrm{Ag}$ (9:1 Ag:Pd sample; Fig. 2 (c)) yielded a blue-shifted 
extinction peak. The blue-shift is consistent with a core-shell Ag-Pd NP structure [22]. It was only for Ag:Pd of 15:1 or lower that the Ag-Pd NP extinction spectrum (Fig. 2 (d)) truly resembled the shape of the Ag seed. A reduction in peak extinction intensity of only $10 \%$ and a blue-shift of $17 \mathrm{~nm}$ was found for a Ag:Pd of 15:1. Due to the high retention of both shape and intensity of the Ag seed's extinction spectrum, the 15:1 Ag-Pd NP were used for photocatalytic testing. A compilation of the respective Ag seed peak extinctions, Ag-Pd NP peak extinctions and peak extinction intensity reductions is given in Table 1. 

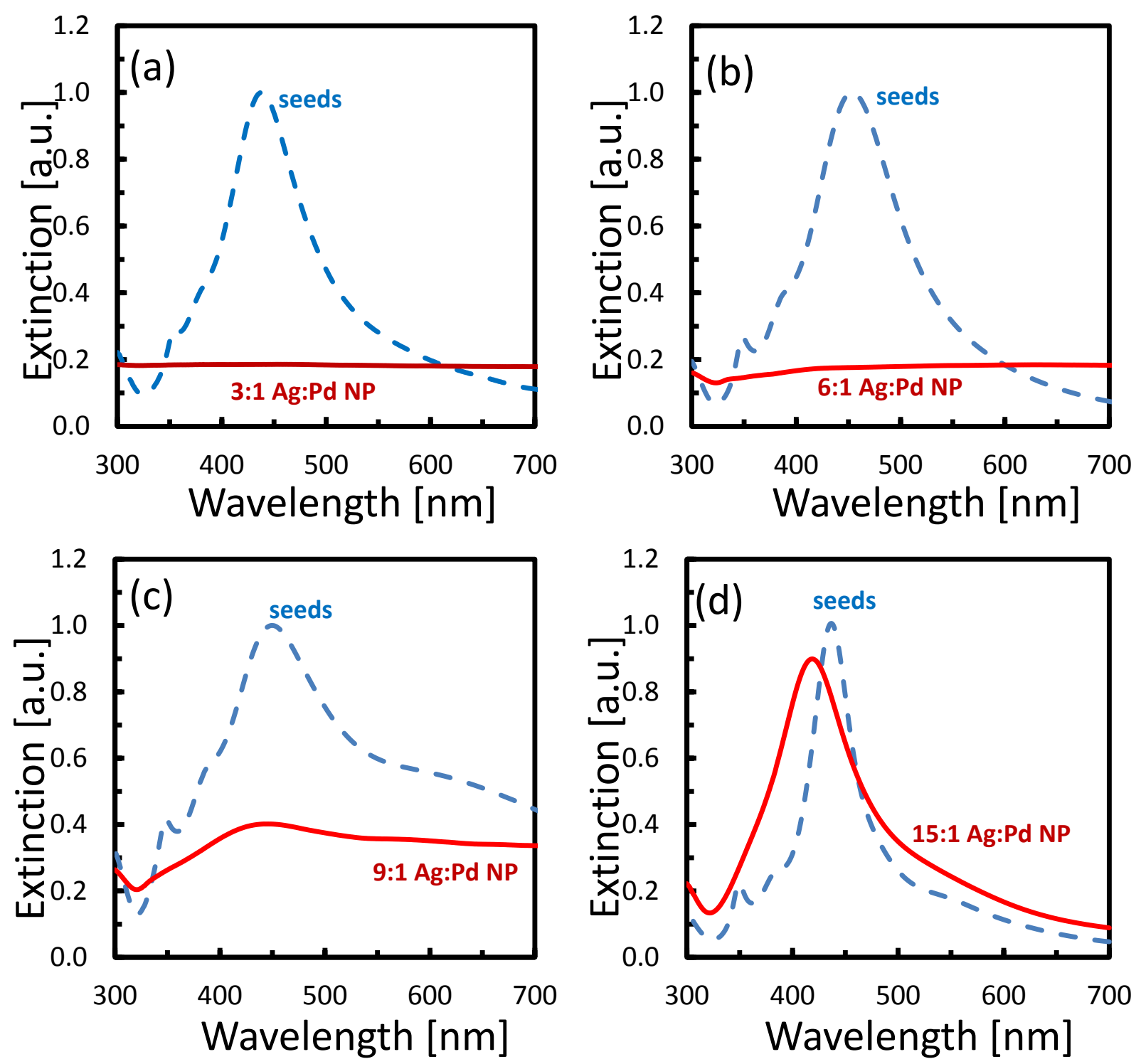

Fig. 2. Extinction spectra of the various Ag-Pd NP and corresponding Ag seeds.

To properly compare the Ag NC and Ag-Pd NP with respect to plasmonic interactions, a batch of $\mathrm{Ag}$ NC was synthesized to best overlap the Ag-Pd NP extinction spectrum in the range at which photons possess the necessary energy to drive the reaction. As shown in Error! Reference source not found., the shoulders, caused by surface plasmon modes of corners and edges [17, 32], follow a similar trend in spectrum for $\lambda \leq 405 \mathrm{~nm}$. This similarity in extinction spectra was used to justify any enhancement found due to the addition of a Pd shell on the $\mathrm{Ag} \mathrm{NC}$ core as enhancement from plasmonic interactions. The extinction spectra of synthesized batches are sensitive to the size and shape distributions of all Ag NPs in 
solution, which results in intensity variations of the seed solutions. The approach here is to study the photocatalytic reactions with equivalent optical activity below $405 \mathrm{~nm}$ for the Ag NCs and Ag-Pd NP at the photon energies relevant for titania photocatalysis.

Table 1. Sample labeling and comparison of Ag seeds and Ag-Pd NP extinction spectra.

\begin{tabular}{|c|c|c|}
\hline Sample label $^{\mathrm{a}}$ & Ag seed peak [nm] & Ag-Pd NP peak [nm] \\
\hline $3: 1$ Ag-Pd NP & 443 & - \\
\hline $6: 1$ Ag-Pd NP & 457 & - \\
\hline 9:1 Ag-Pd NP & 456 & 443 \\
\hline 15:1 Ag-Pd NP & 440 & 423 \\
\hline
\end{tabular}

${ }^{\text {a }}$ Atomic ratio of $\mathrm{Ag}: \mathrm{Pd}$ used for synthesis

An estimated shape distribution of roughly $70-80 \%$ cubes, with residual particles taking on a mixture of spherical and pyramidal shapes, was determined using TEM. Selected TEM images are shown in Fig. , which also reveals a relatively uniform size distribution. The Ag NC yielded an average size of roughly $50 \mathrm{~nm}$. This value agreed very well with that of a plasmonic ruler approach [33], which yielded 44 to $56 \mathrm{~nm}$ edge lengths based on the position of the extinction peak. Analysis also revealed an average shell thickness of $5 \mathrm{~nm}$ for the 9:1 Ag-Pd NP. The average thickness was determined by using the measuring tool on the TEM software, which analyzed both planar and cross-section images. As mentioned previously, electronic surface properties of the Ag NC core should no longer be measurable when the Pd shell thickness exceeds $3 \mathrm{~nm}$, and given that the extinction spectrum of the 15:1 Ag-Pd NP shows little resemblance to its Ag seed's extinction spectrum, the measured shell thickness of $5 \mathrm{~nm}$ is reasonable for the 9:1 Ag-Pd NP. For use the composite materials, the Ag and Ag-Pd NPs were immobilized on P25 TiO2. TEM images are shown in Fig. 4 and additional ones are shown in Fig. S2. In addition to $\mathrm{TiO}_{2}$ planes, the $\mathrm{Ag}$ (111) planes was detected in the Ag sample. Lattice spacing consistent with $\mathrm{AgTi}_{4} \mathrm{O}_{9}$ was also present. Similar observations were made for the $\mathrm{TiO}_{2} / \mathrm{Ag}-\mathrm{Pd}$ sample, with the difference that the $\mathrm{Pd}$ (111) plane was observed. Lattice spacing consistent with $\mathrm{AgPdCl}_{4}$ may also be 
present, but its primary spacing is closely matched to $\mathrm{AgTi}_{4} \mathrm{O}_{9}$. Overall, there did not appear to be substantial differences in the interface between phases in these samples.

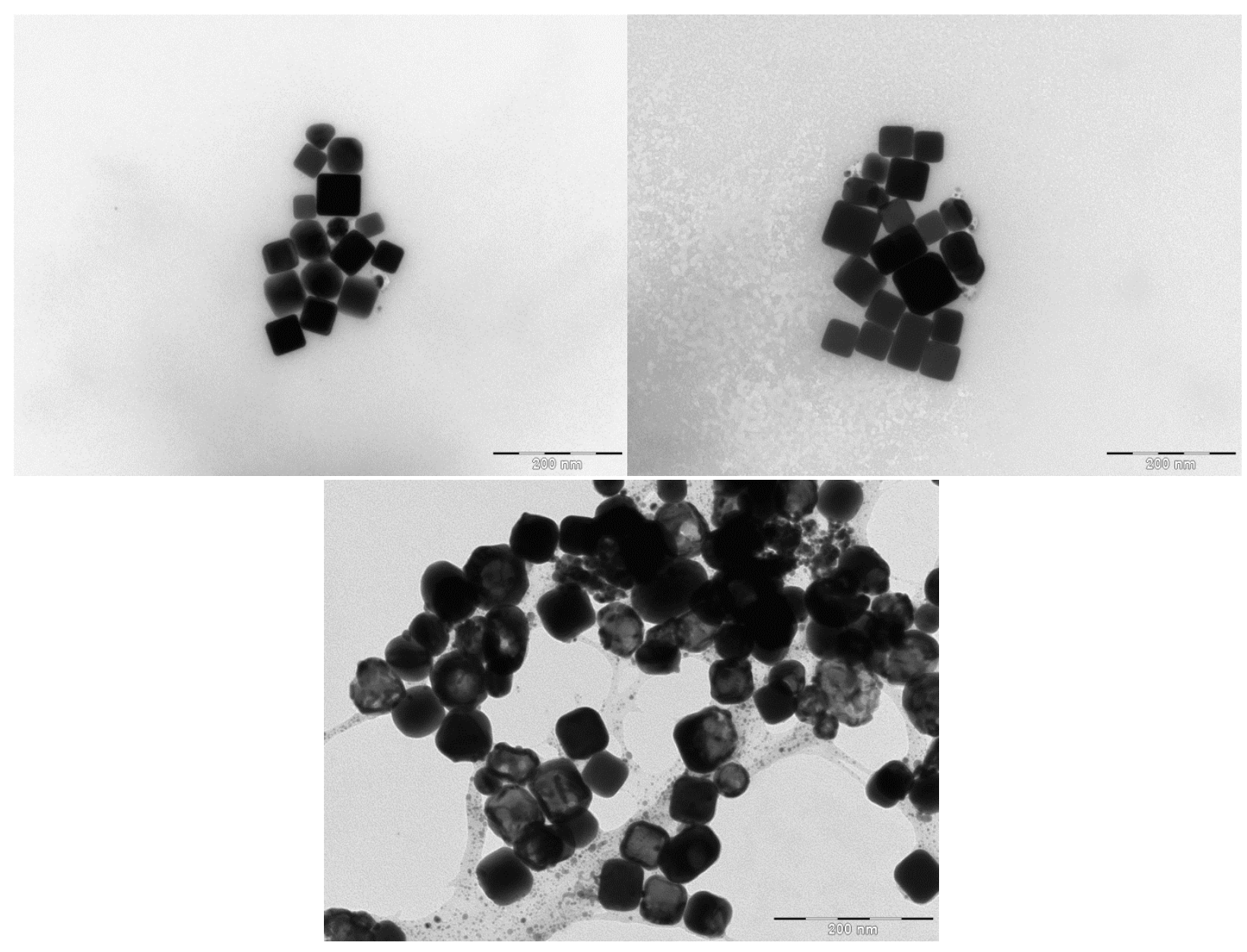

Fig. 3. Selected TEM images of (top) Ag NC and (bottom) Ag-Pd NP (15:1 for Ag: Pd). 


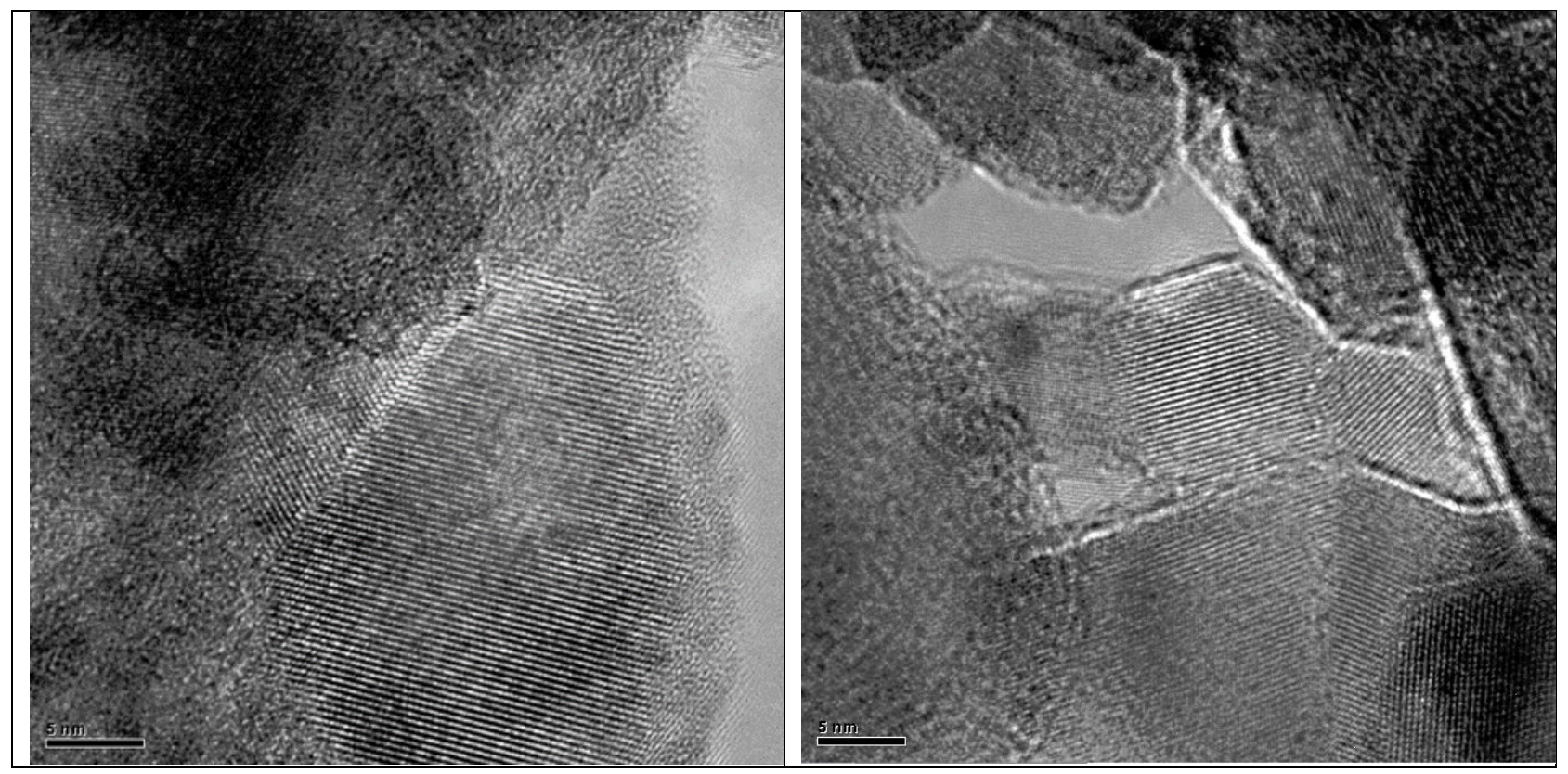

Fig. 4. Selected TEM images of (left) Ag-Pd NP (15:1 for Ag: Pd) and (right) Ag NC and deposited on $\mathrm{TiO}_{2}$. Total metal mass $1 \mathrm{wt} \%$ in each case.

\subsection{Photocatalytic slurry batch reactor}

The photocatalytic degradation of MO under UV-Vis conditions was performed in a shallow dish, batch slurry reactor as a preliminary experiment to investigate the effects of titania/NP (15:1 Ag-Pd NP) composites. As presented in Fig. 5, the addition of Ag NCs and Ag-Pd NPs decreased the MO conversion as compared to $\mathrm{TiO}_{2}$. The conversion decreased with increasing $\mathrm{Ag}$ mass in the catalyst. Even the small $0.3 \mathrm{wt} \%$ loading of $\mathrm{Ag} \mathrm{NC}$ onto $\mathrm{TiO}_{2}$ decreased the observed initial degradation rate of pure titania, -2.7 $\mathrm{E}-5 \mathrm{~mol} \mathrm{MO} /(\mathrm{g} * \mathrm{~s})$, by $28 \%$. Further increases in the $\mathrm{Ag} \mathrm{NC}$ mass loading to 1.0 and $10 \mathrm{wt} \%$ resulted in even worse initial degradation rates of $-1.8 \mathrm{E}-5 \mathrm{~mol} \mathrm{MO} /\left(\mathrm{g}^{*} \mathrm{~s}\right)$ and $-1.1 \mathrm{E}-5 \mathrm{~mol} \mathrm{MO} /\left(\mathrm{g}^{*} \mathrm{~s}\right)$, respectively. The apparent quantum yields are reported in Table 2. These behaviors are consistent with the results of Mogal et al [20], who reported enhancement only for samples with low Ag loadings where Ag oxidized, and Barakat et al $[34,35]$, who reported enhancements for $\mathrm{TiO}_{2}$ with $\mathrm{Pt}$ and $\mathrm{Ag}-\mathrm{Pt}$ but not Ag doping. The primary difference in the current study is the size of the NPs are much larger (e.g., $50 \mathrm{~nm}$ rather than $~ 3$ $\mathrm{nm}[36])$ which would decrease the propensity of surface oxidation and alter the amount of the $\mathrm{TiO}_{2}$ surface exposed (which also depends on metal loading). The decreases in conversion and quantum yields 
were not caused by a decrease in the amount of $\mathrm{TiO}_{2}$ in the reactor as the experiments were performed on an equal $\mathrm{TiO}_{2}$ basis $(1 \mathrm{~g} / \mathrm{L})$.

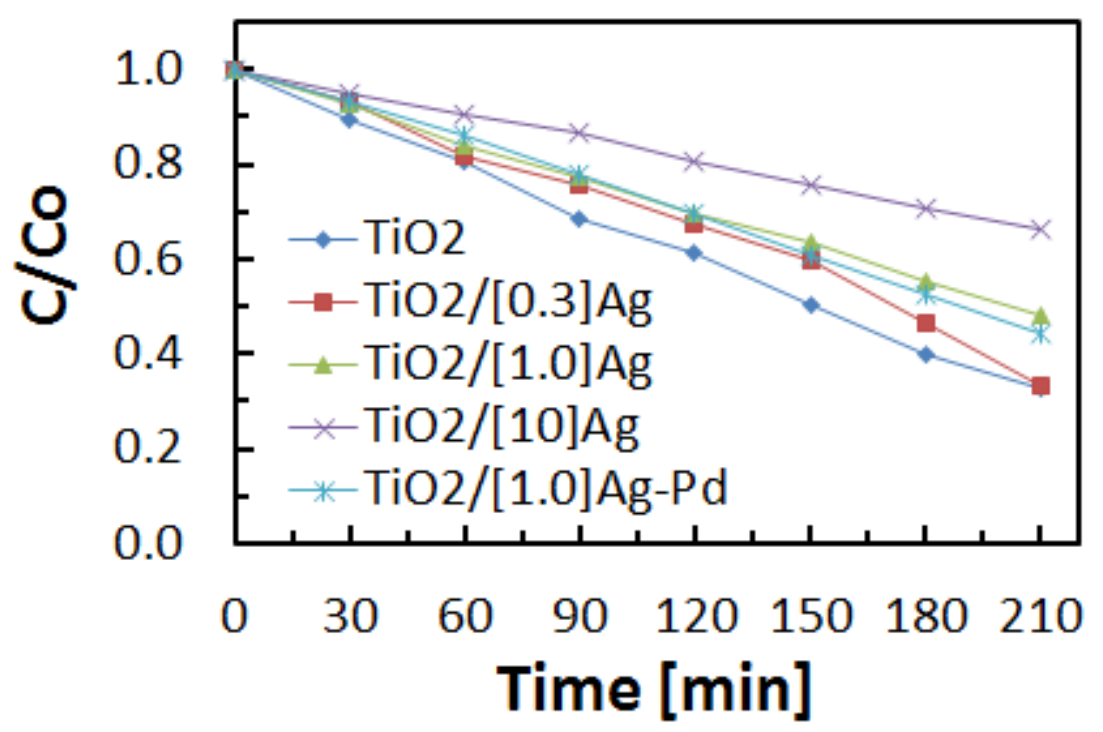

Fig. 3. MO degradation under UV-Vis conditions for $\mathrm{TiO}_{2} / \mathrm{NP}$ composites (with total metal mass loading weight percent in brackets) in a batch slurry reactor.

This difference in experimental findings attributed to the titania-NP composite arrangement could have been caused by low effectiveness of mixing between titania particles in contact with Ag NCs and pure titania particles, light being scattered away from the solution by Ag NCs near the surface of the reaction slurry, and Ag NCs covering up catalytic sites on the surface of titania particles. Regardless, an improvement to the observed reaction rate of the $\mathrm{TiO}_{2} /[1] \mathrm{Ag} \mathrm{NC}$ composite did occur by adding a layer of Pd to the Ag NCs. As seen in Fig. 3, the reaction was still not superior to pure titania, but it is valuable to this study that an improvement of $5 \%$ was seen by the $\mathrm{TiO}_{2} /[1] \mathrm{Ag}-\mathrm{Pd} \mathrm{NP}$ composite when compared to the $\mathrm{TiO}_{2} /[1] \mathrm{Ag} \mathrm{NC}$ composite and the fact this is difference is similar to the relative error is acknowledged, but it is noted that the value is much higher than the $10 \% \mathrm{Ag} \mathrm{NC}$ value. Contributing factors will be discussed later. 
Table 2. Apparent quantum yields for $\mathrm{MO}$ degradation $\left(\Phi_{\mathrm{MO}}\right)$ of $\mathrm{TiO}_{2} / \mathrm{NP}$ composites under UV-Vis conditions in a batch slurry reactor.

\begin{tabular}{|c|c|}
\hline Sample Label & $\Phi_{\mathrm{MO}}[\%]$ \\
\hline $\mathrm{TiO}_{2}$ & 0.029 \\
\hline $\mathrm{TiO}_{2} /[0.3] \mathrm{Ag} \mathrm{NC}$ & 0.023 \\
\hline $\mathrm{TiO}_{2} /[1] \mathrm{Ag} \mathrm{NC}$ & 0.020 \\
\hline $\mathrm{TiO}_{2} /[10] \mathrm{Ag} \mathrm{NC}$ & 0.012 \\
\hline $\mathrm{TiO}_{2} /[1] \mathrm{Ag}-\mathrm{Pd} \mathrm{NP}$ & 0.021 \\
\hline
\end{tabular}

\subsection{Photocatalytic recirculating batch reactor}

In hopes of achieving improved degradation rates compared to pure $\mathrm{TiO}_{2}$ and, with the hypothesis that the component configuration is important to exploit the plasmonic optical properties, a layered arrangement of the titania-NP system was implemented for photocatalytic testing using the improved experimental design. The use of a layered plasmonic layer under the photocatalytic semiconductor reveals a novel methodology to engineer photon pathways in photocatalytic reactors. The multi-layer $\mathrm{TiO}_{2}+\mathrm{NP}$ arrangement showed improved degradation rates for both $\mathrm{TiO}_{2}+\mathrm{Ag} \mathrm{NC}$ and $\mathrm{TiO}_{2}+\mathrm{Ag}-\mathrm{Pd} \mathrm{NP}$ systems. From Fig. 4, the addition of a base layer of Ag NC to the pure titania substrate doubled the degradation rate from $-2.6 \mathrm{E}-5 \mathrm{~mol} \mathrm{RB} /\left(\mathrm{cm}^{2} * \mathrm{~s}\right)$ to $-5.2 \mathrm{E}-5 \mathrm{~mol} \mathrm{RB} /\left(\mathrm{cm}^{2 *} \mathrm{~s}\right)$. However, the addition of a Ag-Pd NP base layer only resulted in an improvement to $-3.4 \mathrm{E}-5 \mathrm{~mol} \mathrm{RB} /\left(\mathrm{cm}^{2} * \mathrm{~s}\right)$. The rate constants from a pseudo-first order kinetic analysis normalized per illuminated surface area were calculated, and the values are reported in Table 3. This decrease in overall enhancement was most likely evidence that, even though the Ag NC and Ag-Pd NP have similar extinction intensities at $\leq 405 \mathrm{~nm}$, the higher scattering efficiency of Ag must have an effect on the NP layer's ability to locally intensify the flux of UV photons in the titania top layer. In addition, Pd may impose some other function (electron trapping, catalytic) when in direct contact with the $\mathrm{TiO}_{2}$. This interface is minimized in the film arrangement and may be connected to why the $\mathrm{TiO}_{2} /[1] \mathrm{Ag}-\mathrm{Pd} \mathrm{NPs}$ showed higher conversion than the $\mathrm{TiO}_{2} /[1] \mathrm{Ag}$ NCs in the batch slurry reactor (Fig. 5), but not in the layered arrangement. The fact that the interface between the Pd shell and titania particles was no longer evenly spread throughout the titania and is concentrated to one continuous titania-Pd interface also seemed to have an effect on the improvements seen in the MO experiments[37]. 


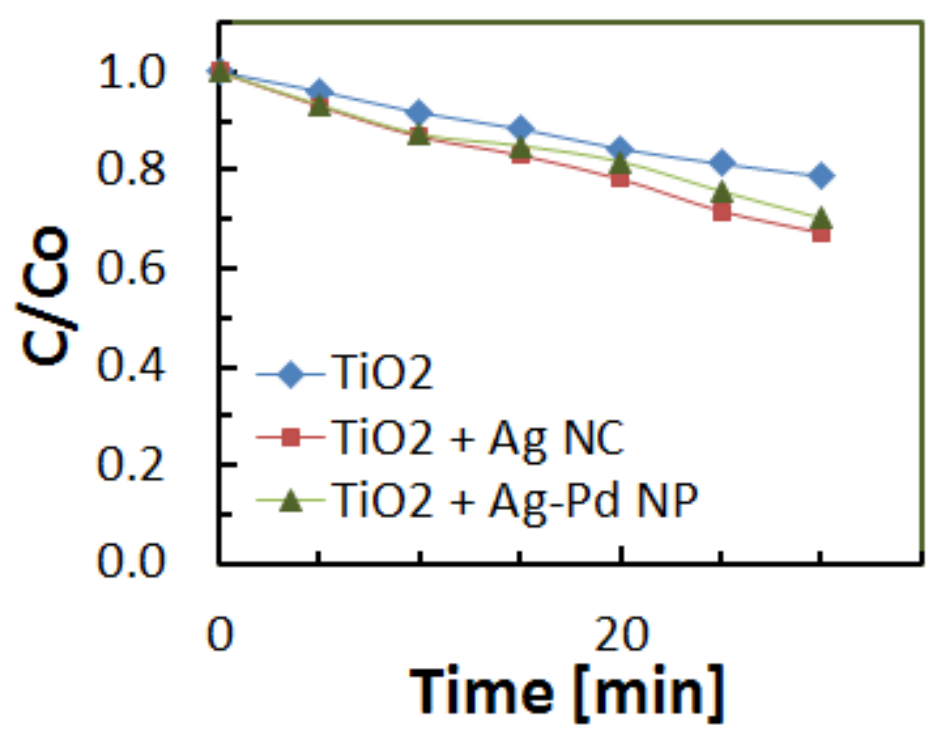

Fig. 4. RB degradation under UV conditions for $\mathrm{TiO}_{2}+\mathrm{NP}$ films in a recirculating batch reactor.

Table 3. Rate constants (k" uv) of $\mathrm{TiO}_{2} / \mathrm{NP}$ composite films and $\mathrm{TiO}_{2}+\mathrm{NP}$ films under UV conditions in a recirculating batch reactor. Pseudo-first order kinetics normalized per illuminated area applied.

\begin{tabular}{|c|c|}
\hline Sample Label & $\mathrm{k}^{\prime}{ }_{\mathrm{UV}}\left[\mathrm{mL} /\left(\mathrm{cm}^{2}{ }^{*} \mathrm{~s}\right)\right]$ \\
\hline $\mathrm{TiO}_{2}$ & 0.0089 \\
\hline $\mathrm{TiO}_{2}+\mathrm{Ag} \mathrm{NC}$ & 0.018 \\
\hline $\mathrm{TiO}_{2}+\mathrm{Ag}-\mathrm{Pd} \mathrm{NP}$ & 0.015 \\
\hline $\mathrm{TiO}_{2} /[0.05] \mathrm{Ag} \mathrm{NC}$ & 0.0091 \\
\hline $\mathrm{TiO}_{2} /[0.1] \mathrm{Ag} \mathrm{NC}$ & 0.010 \\
\hline $\mathrm{TiO}_{2} /[0.05] \mathrm{Ag}-\mathrm{Pd} \mathrm{NP}$ & 0.010 \\
\hline $\mathrm{TiO}_{2} /[0.1] \mathrm{Ag}-\mathrm{Pd} \mathrm{NP}$ & 0.010 \\
\hline
\end{tabular}

To complete this study, $\mathrm{TiO}_{2} / \mathrm{NP}$ composites were testing in the layered film arrangement and the transient profiles from the recirculating batch reactor are presented in Figure 7. The pseudo-first order rate constants normalized per illuminated area are listed in Table 3. Though there was some enhancement relative to pure $\mathrm{TiO}_{2}$ for the various mass loading amounts, it was small and not above the error confidence levels. The mass loading of $0.05 \% \mathrm{Ag} \mathrm{NC}$ did not have an effect on the degradation rate. However, at this mass loading, Ag-Pd NP improved the initial degradation rate to $-2.9 \mathrm{E}-5$ mol $\mathrm{RB} /\left(\mathrm{cm}^{2 *} \mathrm{~s}\right)$. A further increase in the metal mass loading to $0.1 \mathrm{wt} \%$ resulted in an interesting outcome in that the improvement obtained by both the $\mathrm{TiO}_{2} /[0.1] \mathrm{Ag} \mathrm{NC}$ and $\mathrm{TiO}_{2} /[0.1] \mathrm{Ag}-\mathrm{Pd} \mathrm{NP}$ composites fell within error of one another but offered no additional enhancement upon what was provided by the 
$\mathrm{TiO}_{2} /[0.05] \mathrm{Ag}-\mathrm{Pd} \mathrm{NP}$ composite. Based on the earlier results, higher mass loadings of metal in the composites were not tested.

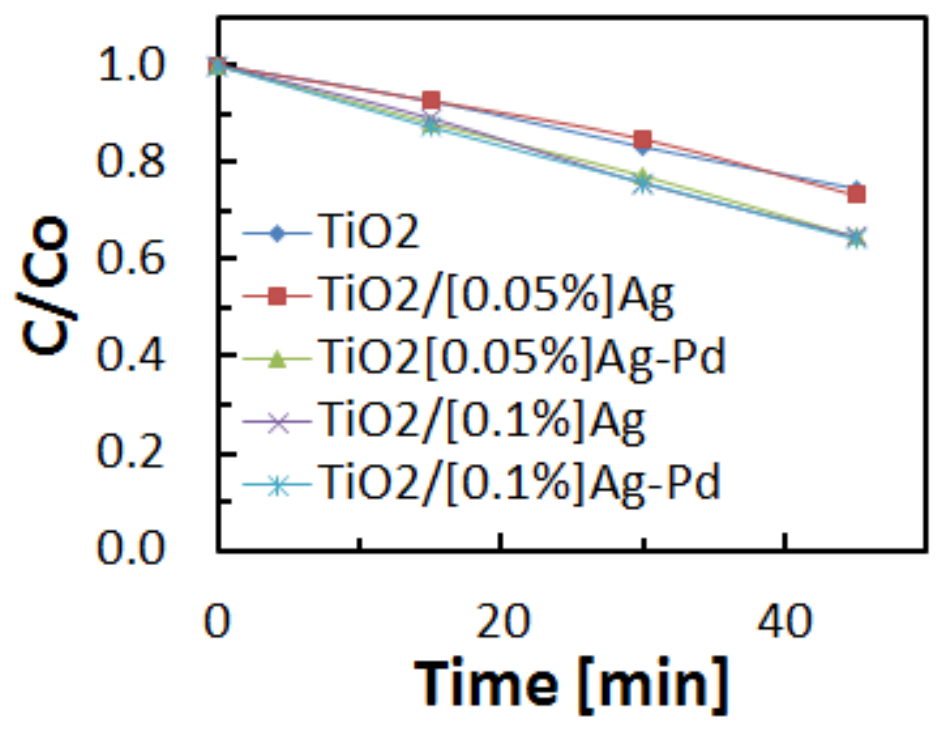

Fig. 5. RB degradation under UV conditions for $\mathrm{TiO}_{2} / \mathrm{NP}$ composite films in a recirculating batch reactor.

\section{Discussion}

The results of this study indicate the importance of configurational effects of plasmonic phenomena in photocatalytic reactors. With $\mathrm{TiO}_{2} / \mathrm{Ag}$ composite catalysts in a batch slurry reactor, performance became worse than the pristine $\mathrm{TiO}_{2}$. These results are consistent to literature $[20,34,35]$ in which various explanations are given and include blockage of active $\mathrm{TiO}_{2}$ surface sites[20], prevention of light absorption[20], and oxidation of $\mathrm{Ag}$ by contact with $\mathrm{TiO}_{2}[10]$. With large $\mathrm{Ag}(75-90 \mathrm{~nm})$ rods, cubes, and spheres demonstrating a maximum enhancement by a factor of nearly 4 (15 wt \% $\mathrm{Ag}$ spheres/ $\mathrm{TiO}_{2}$ ) compared to pristine $\mathrm{TiO}_{2}$ in a solid-state reactor, the enhancements are justified by minimal direct contact between the plasmonic and semiconductor phases and the separation of the two phases by polymeric stabilizers used for the synthesis of the Ag particles [12]. Thus, both the Ag particle size and the use of stabilizers (either inorganic [10]or organic [12]) may be used to minimize the direct interfacial areas between the phases. However, both of these factors are coupled to the tuning of the 
plasmonic resonance [38] and organic separation layer may not be stable under oxidizing conditions [39]. Therefore, geometric configuration is the focus of the current contribution. The use of a recirculating thin film photoreactor permitted the use of layered films rather than composites materials as is the case for a slurry photoreactor.

The layered film approach in the recirculating thin film photoreactor resulted in up to a factor of 2 improvement whereas control experiments with composites film catalysts only showed $\sim 10 \%$ improvement. In the layered arrangement, all explanations mentioned above (blockage of active $\mathrm{TiO}_{2}$ surface sites prevention of light absorption, and oxidation of $\mathrm{Ag}$ by contact with $\mathrm{TiO}_{2}$ ) for decreased performance relative to pristine $\mathrm{TiO}_{2}$ are minimized and, thus, all catalyst films have result in an improvement relative to pristine $\mathrm{TiO}_{2}$. This arrangement permits both maximizing photocatalyst area per illuminated area and photocatalyst absorption area in metal modified photocatalysts, as well as providing a realistic reactor configuration for flow experiments. Since the incorporation of Pd to the Ag phase did not result in improvements in the recirculating thin film photoreactor, the result is consistent with minimum direct contact between phases with a design criteria for plasmonic photocatalytic reactors. Future efforts should focus on simultaneously modifying the semiconductor phase electronically without impacting the light absorption area while also tuning the underneath plasmonic layer for properties specific to photon energies of interest. Innovative ways to increase photon fluxes in photocatalytic reactors are needed to alleviate the challenges of reactor photocatalytic scaling in two-dimensions rather than the typical 3 dimensions for thermo-chemical catalysis.

\section{Conclusions}

Various loadings of Ag NCs and Ag-Pd NPs were studied in $\mathrm{TiO}_{2} / \mathrm{NP}$ composites and $\mathrm{TiO}_{2}+\mathrm{NP}$ layered systems. Experimental procedures were improved upon in that bench scale reactions were conducted in a manner relevant to industrial application. Using the comparison of extinction spectra to optimize the novel Ag-Pd NP synthesis technique, a $\mathrm{Ag}: \mathrm{Pd}$ of 15:1 was determined to result in proper 
retention of the Ag core's distinctive plasmonic properties after addition of a thin Pd shell. Efforts were taken to best distinguish enhancement brought on due to Pd's electron scavenging properties by comparing a set of Ag NCs and Ag-Pd NPs which showed similar extinction trends at $\lambda \leq 410 \mathrm{~nm}$ but it was found that Ag's high scattering efficiency resulted in a greater enhanced degradation rate when compared to Ag-Pd NP in a $\mathrm{TiO}_{2}+\mathrm{NP}$ layered arrangement. To expand upon this, the two shoulders located to the left of the peak of the Ag NC's extinction spectrum are representative of distinctive surface plasmon modes located at the edges and corners of the Ag NC. However, these are not present in the 15:1 Ag-Pd NP extinction spectrum and because these shoulders appear in an area of the spectrum where all light has the potential to generate an exciton, removal of these geometrically specific plasmon modes resulted in a decrease in enhancement brought on by plasmonic interactions with light. The more interesting results were obtained from the $\mathrm{TiO}_{2} / \mathrm{NP}$ composite experiments which took place in the batch type reactor. For the first time, improved photocatalytic degradation performance was shown for a $\mathrm{TiO}_{2} / \mathrm{Ag}-\mathrm{Pd} \mathrm{NP}$ composite and the experiments were conducted in a way relevant to wastewater remediation. Further optimization of these reactor design features and material structure and composition will allow for the most effective utilization of plasmonics in photocatalysis.

\section{Acknowledgements}

Support from NSF grants EEC-1301054 and CBET-1335817 is gratefully acknowledged. Partial support from the Office of Undergraduate Research at USF and the Florida Department of Agriculture and Consumer Services, Division of Aquaculture is also acknowledged. The authors thank Dr. Yusuf Emirov, Samuel Batchelder, Jun Liu, and Andrew Orbeck for assistance in collection of experimental data.

\section{References}

[1] S. Ahmed, M.G. Rasul, W.N. Martens, R. Brown, M.A. Hashi, Heterogeneous photocatalytic degradation of phenols in wastewater: A review on current status and developments, Desalination, 261 (2010) 3-18. 
[2] M.R. Hoffmann, S.T. Martin, W.-I. Choi, D.W. Bahnemann, Environmental Applications of Semiconductor Photocatalysis, Chem. Rev., 95 (1995) 69-96.

[3] A. Fujishima, T.N. Rao, D.A. Tryk, Titanium dioxide photocatalysis, Journal of Photochemistry and Photobiology C: Photochemistry Reviews, 1 (2000) 1-21.

[4] J.-M. Herrmann, Heterogeneous photocatalysis: fundamentals and applications to the removal of various types of aqueous pollutants, Catalysis Today, 53 (1999) 115-129.

[5] B. Liu, X. Zhao, C. Terashima, A. Fujishima, K. Nakata, Thermodynamic and kinetic analysis of heterogeneous photocatalysis for semiconductor systems, Phys. Chem. Chem. Phys., 16 (2014) 8751 -8760.

[6] S. Bai, J. Jiang, Q. Zhang, Y. Xiong, Steering charge kinetics in photocatalysis: intersection of materials syntheses, characterization techniques and theoretical simulations, Chem. Soc. Rev., 44 (2015) 2893-2939.

[7] T. Van Gerven, G. Mul, J. Moulijn, A. Stankiewicz, A review of intensification of photocatalytic processes, Chemical Engineering and Processing, 46 (2007) 781-789.

[8] J. Yang, D. Wang, H. Han, C. Li, Roles of Cocatalysts in Photocatalysis and Photoelectrocatalysis, Acc. Chem. Res., 46 (2013) 1900-1909.

[9] C.J. Pestana, P.K.J. Robertson, C. Edwards, W. Wilhem, C. McKenzie, L.A. Lawton, A continuous flow packed bed photocatalytic reactor for the destruction of 2-methylisoborneol and geosmin utilising pelletised TiO2, Chemical Engineering Journal, 235 (2014) 293-298.

[10] K. Awazu, M. Fujimaki, C. Rockstuhl, J. Tominaga, H. Murakami, Y. Ohki, N. Yoshida, T. Watanabe, A Plasmonic Photocatalyst Consisting of Silver Nanoparticles Embedded in Titanium Dioxide, Journal of American Chemical Society, 130 (2008) 1676-1680.

[11] D.B. Ingram, S. Linic, Water Splitting on Composite Plasmonic-Metal/Semiconductor

Photoelectrodes: Evidence for Selective Plasmon-Induced Formation of Charge Carriers near the Semiconductor Surface, J . Am. Chem. Soc., 133 (2011) 5202-5205.

[12] P. Christopher, D.B. Ingram, S. Linic, Enhancing Photochemical Activity of Semiconductor Nanoparticles with Optically Active Ag Nanostructures: Photochemistry Mediated by Ag Surface Plasmons, J. Phys. Chem. C, 114 (2010) 9173-9177.

[13] X. Ma, Y. Dai, Z. Lou, B. Huang, M.-H. Whangbo, Electron-Hole Pair Generation of the VisibleLight Plasmonic Photocatalyst Ag@ AgCl: Enhanced Optical Transitions Involving Midgap Defect States of AgCl, J. Phys. Chem. C, 118 (2014) 12133-12140.

[14] X.-C. Ma, Y. Dai, L. Yu, B.-B. Huang, Energy transfer in plasmonic photocatalytic composites, Light: Science \& Applications, 5 (2016) e16017.

[15] C. Wang, O. Ranasingha, S. Natesakhawat, P.R. Ohodnicki Jr., M. Andio, J.P. Lewis, C. Matranga, Visible light plasmonic heating of $\mathrm{Au}-\mathrm{ZnO}$ for the catalytic reduction of CO2, Nanoscale, 5 (2013) 69686974.

[16] S. Linic, P. Christopher, H. Xin, A. Marimuthu, Catalytic and Photocatalytic Transformations on Metal Nanoparticles with Targeted Geometric and Plasmonic Properties, Acc. Chem. Res., 46 (2013) 1890-1899.

[17] Q. Zhang, W. Li, L.-P. Wen, J. Chen, Y. Xia, Facile Synthesis of Ag Nanocubes of 30 to $70 \mathrm{~nm}$ in Edge Length with CF3COOAg as a Precursor, Chem. Eur. J., 16 (2010) $10234-10239$.

[18] J. Zeng, C. Zhu, J. Tao, M. Jin, H. Zhang, Z.-Y. Li, Y. Zhu, Y. Xia, Controlling the Nucleation and Growth of Silver on Palladium Nanocubes by Manipulating the Reaction Kinetics, Angewandte Chemie International Edition, 51 (2012) 2354 -2358.

[19] J. Gong, F. Zhou, Z. Li, Z. Tang, Controlled synthesis of non-epitaxially grown Pd@ Ag core-shell nanocrystals of interesting optical performance, Chem. Commun., 49 (2013) 4379-4381

[20] S.I. Mogal, V.G. Gandhi, M. Mishra, S. Tripathi, T. Shripathi, P.A. Joshi, D.O. Shah, Single-Step Synthesis of Silver-Doped Titanium Dioxide: Influence of Silver on Structural, Textural, and Photocatalytic Properties, Ind. Eng. Chem. Res., 53 (2014) 5749-5758.

[21] I. Chorkendorff, J.W. Niemantsverdriet, Concepts of Modern Catalysis and Kinetics, 2nd edition, (2007) Wiley (Weinheim). 
[22] Y. Yang, X. Gong, H. Zeng, L. Zhang, X. Zhang, C. Zou, S. Huang, Combination of Digestive Ripening and Seeding Growth As a Generalized Route for Precisely Controlling Size of Monodispersed Noble Monometallic, Shell Thickness of Core-Shell and Composition of Alloy Nanoparticles, J. Phys. Chem. C, 114 (2010) 256-364.

[23] U. Sanyal, D.T. Davis, B.R. Jagirdar, Bimetallic core-shell nanocomposites using weak reducing agent and their transformation to alloy nanostructures, Dalton Trans., 42 (2013) 7147-7157.

[24] K. Tedsree, T. Li, S. Jones, C. Wong, C.W.A. Chan, K.M.K. Yu, P.A.J. Bagot, E.A. Marquis, G.D.W. Smith, S.C.E. Tsang, Hydrogen production from formic acid decomposition at room temperature using a Ag-Pd core-shell nanocatalyst, Nature Nanotech., 6 (2011) 302-307.

[25] J. Chen, B. Wiley, J. McLellan, Y. Xiong, Z.-Y. Li, Y. Xia, Optical Properties of Pd-Ag and Pt-Ag Nanoboxes Synthesized via Galvanic Replacement Reactions, Nano Lett., 5 (2005) 2058-2062.

[26] S.L. Pettit, C.H. McCane, J.T. Wolan, J.N. Kuhn, Synthesis and Characterization of Composite Photocatalytic Semiconductors (InVO4-TiO2) using Pure and Mixed Phase Titania Powders, Catalysis Letters, 43 (2013) 772-776.

[27] P. Ameta, K. A., M. Paliwal, R. Ameta, R. Malkani, Photocatalytic Bleaching of Rose Bengal by Some Coloured Semiconducting Oxides, Bull. of the Cat. Soc. of Ind., 6 (2007) 130-135.

[28] R. Kumawat, I. Bhati, R. Ameta, Role of some metal ions in in photocatalytic degradation of Rose Bengal dye, Ind. J. Chem. Tech., 19 (2012) 191-194.

[29] N. Mittal, A. Shah, P.B. Punjabi, V.K. Sharma, Photodegradation of Rose Bengal using MnO2 (Manganese Dioxide), Rasayan J. Chem., 2 (2009) 516-520.

[30] S.B. Sharma, R. Ameta, R. Malkani, S. Ameta, Photocatalytic degradation of Rose Bengal using semiconducting zinc sulphide as the photocatalyst, J. Serb. Chem. Soc., 78 (2013) 897-905.

[31] J.W. Pickering, V.R. Bhethanabotla, J.N. Kuhn, Assessment of mechanisms for enhanced performance of $\mathrm{TiO} 2 / \mathrm{YAG}$ : $\mathrm{Yb}+3, \mathrm{Er}+3$ composite photocatalysts for organic degradation, Applied Catalysis B: Environmental, 202 (2017) 147-155.

[32] B.J. Wiley, Y. Chen, J.M. McLellan, Y. Xiong, Z.Y. Li, D. Ginger, Y. Xia, Synthesis and Optical Properties of Silver Nanobars and Nanorice, Nano Lett., 7 (2007) 1032-1036.

[33] Y. Wang, Y. Zheng, C.Z. Huang, Y. Xia, Synthesis of Ag Nanocubes 18-32 nm in Edge Length: The Effects of Polyol on Reduction Kinetics, Size Control, and Reproducibility, Journal of American Chemical Society, 135 (2013) 1941-1951.

[34] M.A. Barakat, R.I. Al-Hutailah, E. Qayyum, J.N. Kuhn, Pt nanoparticles/TiO2 for photocatalytic degradation of phenols in wastewater, Environmental Technology, 35 (2014) 137-144.

[35] M.A. Barakat, R.I. Al-Hutailah, M.H. Hashim, E. Qayyum, J.N. Kuhn, Titania-supported silverbased bimetallic nanoparticles as photocatalysts, Environ Sci Pollut Res, 20 (2013) 3751-3759.

[36] E. Qayyum, V.A. Castillo, K. Warrington, M.A. Barakat, J.N. Kuhn, Methanol Oxidation over Silica-Supported Pt and Ag Nanoparticles: Towards Selective Production of Hydrogen and Carbon Dioxide Catal. Comm., 28 (2012) 128-133.

[37] W. Li, D. Li, Y. Lin, P. Wang, W. Chen, X. Fu, Y. Shao, Evidence for the Active Species Involved in the Photodegradation Process of Methyl Orange on TiO2, J. Phys. Chem. C, 116 (2012) 3552-3560.

[38] C. Burda, X. Chen, R. Narayanan, M.A. El-Sayed, Chemistry and Properties of Nanocrystals of Different Shapes, Chem. Rev., 105 (2005) 1025-1102.

[39] S.O. Blavo, E. Qayyum, L.M. Baldyga, V.A. Castillo, M.D. Sanchez, K. Warrington, M.A. Barakat, J.N. Kuhn, Verification of Organic Capping Agent Removal from Supported Colloidal Synthesized Pt Nanoparticle Catalysts, Topics in Catalysis, 56 (2013) 1835-1842. 


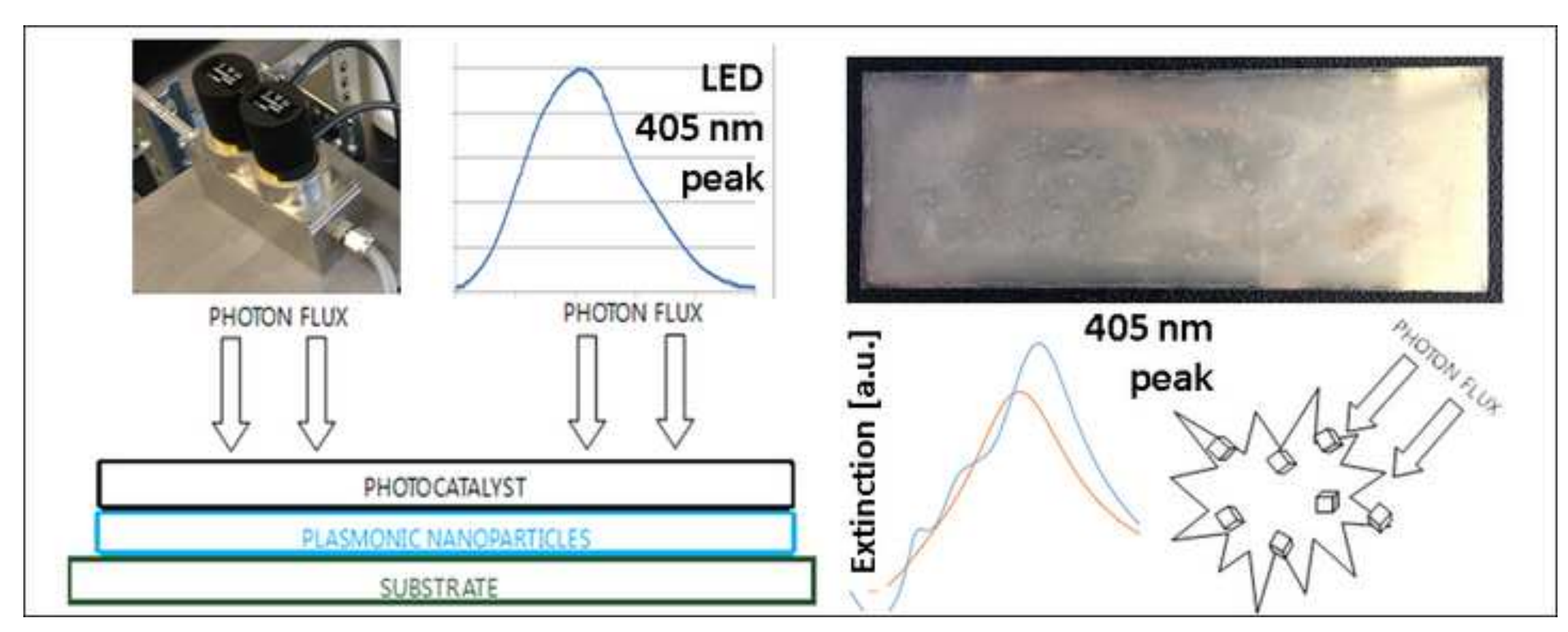

Graphical Abs 